\title{
INNOVACIÓN Y TERRITORIO \\ Una encuesta a pequeñas y medianas empresas industriales
}

\author{
INNOVATION AND TERRITORY
}

A survey of industrial small and medium enterprises

CRIStina Lavía cristina.lavia@ehu.es

Universidad del País Vasco (UPV-EHU). España

BeATRIz Otero beatriz.otero@ehu.es

Universidad del País Vasco (UPV-EHU). España

Mikel OLAzaran mikel.olazaran@ehu.es

Universidad del País Vasco (UPV-EHU). España

Eneka Albizu eneka.albizu@ehu.es

Universidad del País Vasco (UPV-EHU). España

\section{Resumen}

Este trabajo analiza los procesos de innovación en las pequeñas y medianas empresas industriales (Pymes) y, especialmente, las relaciones de cooperación que se establecen en los mismos, desde la perspectiva de los sistemas de innovación. A partir de una encuesta realizada a las Pymes industriales guipuzcoanas, se analizan las características y capacidades internas de innovación de estas organizaciones, sus fuentes externas y las relaciones de cooperación con otras empresas y con agentes de I+D, así como la influencia del entorno regional en los procesos de innovación. Los resultados empíricos obtenidos confirman buena parte de los postulados de la teoría de los sistemas regionales de innovación, especialmente la relativa al llamado "modelo interactivo", aunque también muestran especificidades del caso vasco.

\section{Palabras Clave}

Aprendizaje interactivo, Políticas de innovación, Sistemas regionales de innovación.

\section{ABstract}

This paper analyzes the industrial innovation processes of small and medium enterprises (SMEs) from the point of view of the innovation systems perspective. The focus is on the cooperation relationships which SMEs develop in their innovation processes. Based on a survey to industrial SMEs of the Basque province of Gipúzcoa (Northern Spain), the paper looks at internal innovation capabilities, external sources of innovation, cooperation with other firms and R\&D agents, and influence of the regional environment. The empirical results obtained confirm many or the trends emerging from the international regional innovation systems literature, especially those related to the so-called "interactive model", but they also show some specificities of the Basque case.

\section{KEYWORDS}

Innovation Policies, Interactive Learning, Regional Innovation Systems. 


\section{Sistema regional de innovación, Pymes e innovación*}

\section{El modelo interactivo de innovación}

La perspectiva del "sistema nacional de innovación" (SNI) sostiene que las instituciones sociales condicionan o canalizan la acción económica en general y los procesos de innovación en particular (Lundvall 1992a; Nelson 1993; Edquist 1997). Las instituciones son conjuntos de hábitos, rutinas, reglas, normas y leyes que regulan la relación entre personas y moldean la interacción humana, esto es, "modos prevalentes de hacer las cosas", modos de coordinación multiplicadores de la acción, de cara a obtener un rendimiento deseado en contextos donde intervienen las acciones e interacciones de distintos actores (Johnson 1992; Nelson 2008). Las instituciones reducen la incertidumbre y la búsqueda de información necesaria para la acción individual y colectiva, y son, por tanto, componentes fundamentales de todo sistema social. Para esta perspectiva, el cambio social o socioeconómico es un producto de las relaciones entre la tecnología (o "tecnología física") y las instituciones sociales (o "tecnología social") (Nelson 2008: 269). Dentro de la perspectiva del SNI, la aplicación a nivel regional ("sistema regional de innovación", SRI) ha ganado una gran aceptación en los últimos años (Braczyk et al. 1996; Cooke y Morgan 1998; Storper 1997; Maskell y Malmberg 1999; Cooke, Gómez y Etxeberria 1997; Maskell 2001; Organización para la Cooperación y el Desarrollo Económico 2001). La perspectiva del SRI enlaza con un creciente interés por la importancia del entorno regional de la innovación, especialmente para las pequeñas y medianas empresas (Pymes), y con un mayor peso de las políticas regionales que favorecen y promueven la innovación.

Desde la perspectiva del SRI, la innovación se concibe como un proceso de aprendizaje interactivo dentro de la empresa (comunicación y relaciones entre departamentos, niveles jerárquicos y personas en la organización) y entre ésta y otras organizaciones (clientes y proveedores, institutos de I+D, agencias públicas, etc.) (Lundvall 1992a). El apendizaje interactivo interno y externo tiene lugar dentro de un entorno institucional y cultural donde las relaciones entre el sector público y el privado, el sistema educativo y formativo, el marco de relaciones laborales y el sistema financiero tienen una gran importancia.

Se trata, además, de un proceso localizado, donde factores contextuales específicos pueden promover los procesos de creación y aplicación de conocimiento. Se considera que la aglomeración de empresas supone un ahorro en los costes de transacción en las relaciones entre empresas y favorece las interacciones basadas en la confianza

\footnotetext{
"Este trabajo fue realizado gracias a la ayuda recibida del programa "Red Guipuzcoana de Ciencia, Tecnología e Innovación" (Departamento de Innovación y Sociedad del Conocimiento, Diputación Foral de Gipuzkoa) y del proyecto "Pymes industriales en el sistema regional de innovación" (Ministerio de Ciencia e Innovación, CSO2008-06520-C02-01).
} 
mutua y el intercambio de conocimiento no codificado. La confianza, la reciprocidad, los valores compartidos, las redes y las normas aceleran la transferencia de información y el desarrollo de nuevo conocimiento. Los "fallos del mercado" para el intercambio de conocimiento entre empresas pueden superarse si las relaciones puramente económicas son reemplazadas por acuerdos de intercambio recíprocos y estables basados en la confianza. Las redes de colaboración interempresarial son particularmente importantes en el caso de las Pymes, que conforman la gran mayoría del tejido empresarial europeo. El modelo interactivo parte de la premisa de la importancia fundamental del conocimiento tácito (y sus procesos de creación y transformación) en la innovación, así como de su carácter específico, dependiente del contexto social. La proximidad geográfica adquiere así una importancia esencial en el surgimiento de capacidades de innovación (Asheim y Gertler 2005:309-310). La noción de aprendizaje interactivo interempresarial entre productor y usuario de Lundvall y equipo es especialmente relevante a este respecto (Lundvall 1992b). Según estos autores, la importancia de los sistemas de innovación se deriva del hecho de que los mercados están "organizados" de maneras diferentes en regiones o entornos institucionales distintos.

Las innovaciones de producto tienden a producirse dentro de unas relaciones productor-usuario que van más allá de la racionalidad instrumental 0 , dicho de otra manera, trascienden a las puras relaciones de mercado. Las relaciones de mercado están caracterizadas por el intercambio de información cualitativa, la cooperación, la existencia de una relación más o menos jerárquica y la confianza mutua entre un número reducido de participantes.

Por medio de la relación estable entre usuario y productor se comunica información sobre oportunidades tecnológicas y necesidades del usuario. Productor y usuario desarrollan un código común de comunicación que facilita el intercambio de información y la adaptación de las nuevas tecnologías a las necesidades de los usuarios avanzados. Las relaciones usuario-productor pueden ser jerárquicas o de poder, pero debe haber en ellas un elemento de lealtad, confianza y respeto a la autonomía de las partes (Lundvall 1992b: 52). Lundvall y colegas destacan la importancia de los mercados organizados frente a la integración vertical donde, si bien los costes de transacción entre las partes son menores, en el largo plazo se pierden las ventajas del aprendizaje interactivo entre un abanico más amplio de productores y usuarios independientes.

El modelo interactivo e incremental de innovación es especialmente característico en entornos donde prima el conocimiento específico o sintético o en sectores como la ingeniería mecánica y la máquina-herramienta (Asheim y Coenen 2005; Asheim y Gertler 2005; Breschi y Malerba 1997; Malerba 2004; Olazaran, Albizu y Otero 2009). En los sectores en los que el conocimiento sintético es dominante los procesos de innovación se basan en nuevas combinaciones de elementos existentes, frecuentemente en interacción con clientes (usuarios) o proveedores. Se trata de procesos incrementales de desarrollo de productos o procesos, dirigidos a solucionar problemas o necesidades de los clientes. En estos procesos inductivos de experimentación y desarrollo el conocimiento tácito, el know-how concreto y las capacidades prácticas tienen una gran importancia. Los 
procesos de innovación son por tanto diferentes a aquellos procesos en los que prima el conocimiento "analítico" (científico, formalizable, codificable, patentable, publicable), típico de nuevos sectores como la biotecnología o las tecnologías de la información (Asheim y Coenen 2005; Asheim y Gertler 2005).

El modelo interactivo de aprendizaje (learning by doing, by using, by interacting) es especialmente relevante para el estudio de la innovación en el País Vasco, donde prima el desarrollo incremental de productos y la colaboración interempresarial (con un número reducido de organizaciones) en sectores de intensidad tecnológica media, con especial presencia de la fabricación de bienes de equipo (Olazaran, Albizu y Otero 2009). El sistema vasco de innovación se situaría así dentro de la tipología de "sistema territorialmente imbricado" (territorially embedded regional innovation system, Asheim y Gertler 2005:300), caracterizado por procesos locales de aprendizaje basados en la proximidad geográfica, social y cultural, sin una relación intensa con organizaciones productoras de conocimiento. Las políticas regionales se han dirigido a estimular la relación entre Pymes y centros tecnológicos, algo que, como veremos, se ha conseguido en buena medida. No obstante, la fase de "enriquecimiento" (por decirlo de alguna manera) de las capacidades tecnológicas regionales hacia un modelo con una base científica más fuerte (donde las organizaciones productoras de conocimiento jueguen un papel más decisivo) está todavía en un estadio inicial (Orkestra 2009).

\section{Pymes industriales en el sistema regional de innovación}

Recientemente se han realizado diferentes estudios desde la perspectiva de sistemas de innovación que se centran en la influencia del entorno regional en la actividad innovadora de las Pymes. Estos estudios ofrecen conclusiones interesantes sobre la especificidad de los procesos de innovación en estas organizaciones, las fuentes externas y las relaciones de cooperación que establecen en sus procesos de innovación, así como la influencia del entorno regional en los procesos de innovación de las Pymes.

Se subraya que, para la mayoría de las Pymes, la innovación es un proceso que se asienta principalmente sobre bases internas de conocimiento (Kaufman y Tödtling 2000; Freel 2003; Freel y Harrison 2006; Gebauer et al. 2005). Las Pymes poseen una base limitada de recursos, lo cual limita sus posibilidades de realizar actividades de investigación y desarrollo (I+D) y de establecer relaciones de cooperación con otros agentes. Entre las razones de estas limitaciones se encuentran la falta de recursos financieros y de personal, la falta de tiempo, falta de "know how tecnológico", y falta de capacidades para la búsqueda y selección de información relevante del exterior (Kaufmann y Tödtling 2002; Smallbone et al. 2003). En este contexto se subraya la importancia de que las empresas hayan desarrollado competencias internas como condición indispensable para que puedan establecer relaciones fructíferas de cooperación con agentes externos y sean capaces de hacer uso de información externa e integrarla con la procedente del interior de la empresa (Kauffman y Tödtling 2000; Koschatzky y Sternberg 2000; Freel y Harrison 2006). 
Debido a su menor capacidad para moldear e influenciar el entorno externo, la innovación se produce de manera reactiva, como respuesta a circunstancias cambiantes del entorno, y no forma parte de una estrategia proactiva a largo plazo (Freel 2000; Hassink 1997; Smallbone et al. 2003). Este carácter reactivo y a corto plazo de la innovación en Pymes dificulta la cooperación con agentes regionales de I+D (universidades, centros tecnológicos), los cuales configuran su agenda de investigación a medio/ largo plazo (Hassink 1997). Por otro lado, la innovación en Pymes tiene un marcado carácter market pull (Grotz y Braun 1997; Doloreux 2003; Kaufman y Tödtling 2002). Los estudios coinciden en señalar el papel tractor del cliente en los procesos de innovación de las Pymes y cómo, muchas veces, éstas se ven impulsadas por sus clientes a realizar nuevos desarrollos tecnológicos (Hassink 1997; Kauffman y Tödtling 2002; 2003; Gebauer et al. 2005).

Por tanto, los estudios realizados desde el prisma SRI prestan especial atención al análisis de las relaciones de cooperación que establecen las Pymes en sus procesos de innovación y al ámbito geográfico en que éstas se circunscriben. El hecho de que la mayoría de las innovaciones sea market pull tiene como consecuencia que las redes de cooperación para muchas Pymes se limite a relaciones con agentes dentro de la cadena de valor, clientes y proveedores (Freel 2000; 2003; Doloreux 2003). Como señalan Kaufman y Tödtling (2002) unas pocas relaciones duraderas y selectivas con estos agentes moldean la mayor parte de las actividades de innovación de las Pymes. Esto es especialmente cierto para empresas que realizan innovaciones incrementales en sectores maduros, pero también para empresas con desarrollos basados en avances científicos (Freel 2003; Hassink 1997). Frecuentemente, estas relaciones son de carácter informal (Fritsch 2001) y, en ellas, el precio no es el único factor determinante en una operación comercial. También la existencia de valores compartidos, normas comunes y confianza entre las partes resultan de vital importancia (Doloreux 2003; 2004; Asheim y Coenen 2005; Hassink 1997; Grotz y Braun 1997).

Fuera de la cadena de valor, los vínculos con universidades y agentes de I+D son mucho menores en frecuencia o intensidad (Grotz y Braun 1997; Hassink 1997; Koschatzky y Sternberg 2000; Freel 2000; Kaufmann y Tödtling 2002; Doloreux 2003; 2004; Gebauer et al. 2005). La cooperación con estos agentes se circunscribe a empresas más grandes y/o con mayores capacidades tecnológicas (Koschatzky y Zenker 1999; Kaufmann y Tödtling 2002). Hassink (1997) destaca que las empresas con mayores debilidades en tecnología, que deberían ser el grupo objetivo de estos agentes, tienden a ignorar estas infraestructuras de $\mathrm{I}+\mathrm{D}$, mientras que las empresas con mayores capacidades en I+D e innovación son las que recurren a estas infraestructuras con mayor frecuencia. Asímismo, el carácter reactivo de las innovaciones en Pymes dificulta la colaboración con agentes de I+D, que poseen horizontes de investigación a más largo plazo.

A pesar de las limitaciones de las denominadas "políticas de oferta" (creación de infraestructuras de transferencia de tecnología) que ponen de manifiesto estos estudios, se enfatiza que la existencia de un entorno regional que favorezca la actividad innovadora es especialmente importante en el caso de las Pymes, ya que éstas se encuentran más imbricadas en el entorno regional que las empresas más grandes (Fritsch 2001; 
Koschatzky y Sternberg 2000; Koschatzky y Zenker 1999; Kauffmann y Tödtling 2002). En este sentido, son relevantes las conclusiones del estudio realizado por Copus et al. (2008) en 12 regiones (centrales y periféricas) pertenecientes a 6 países europeos sobre la importancia del entorno regional en las actividades innovadoras de las Pymes. Concluyen que las diferencias interregionales en las tasas de innovación son la consecuencia de heterogeneidad regional y no de características observables de las empresas.

Finalmente, estos estudios destacan que la región es especialmente importante para el intercambio de conocimiento tácito y para la provisión de mano de obra cualificada, ambos son mecanismos muy importantes en los procesos de innovación de las empresas pequeñas (Asheim y Coenen 2005; Kauffmann y Tödtling 2003; Grotz y Braun 1997; Doloreux 2003; Gebauer et al. 2005).

\section{Planteamiento del trabajo empírico}

\section{Objetivo e hipótesis}

El objetivo general de este estudio es caracterizar las relaciones de cooperación de las Pymes guipuzcoanas en materia de innovación, siendo sus objetivos específicos los siguientes: a) analizar cuáles son los principales partners de la cooperación y describir las características de estas relaciones; b) analizar la influencia del entorno regional en las actividades innovadoras de las empresas; $y, c$ ) analizar la influencia del tamaño empresarial en los patrones de cooperación entre las Pymes guipuzcoanas y los agentes externos.

Las hipótesis que se van a someter a contraste son cuatro, a saber:

H1: El modelo interactivo de aprendizaje, basado en la cooperación entre los actores, es la base de los procesos de innovación.

H2: El Sistema Regional de Innovación facilita los procesos de innovación en las Pymes industriales guipuzcoanas.

H3: La proximidad geográfica es un factor determinante en el surgimiento de capacidades de innovación.

H4: La capacidad interna de innovación de las Pymes influye en gran medida en sus patrones de cooperación.

\section{Metodología}

Los datos analizados proceden de una encuesta telefónica a 147 Pymes industriales guipuzcoanas realizada en noviembre de 2008 , siendo la duración del trabajo de campo de dos semanas. Para la identificación del entrevistado/a en cada empresa escogida, esto es, la selección de la unidad muestral, se ha empleado combinadamente el criterio de orden jerárquico y especialidad funcional. De esta manera los informantes han sido el director gerente, el director de I+D o el responsable de la Oficina Técnica. 
Las empresas fueron seleccionadas aleatoriamente de una población total de 454 empresas de 25 a 249 empleados radicadas en el territorio de Gipúzcoa y estratificadas por tamaño (Base de datos SABI de Bureau Van Dick 2008). La muestra global implica un error muestral de $\pm 6,6 \%$ en estimaciones para un NC $95 \%$ y en el supuesto de variabilidad más desfavorable $(p=q=0,5)$. La tasa de rechazo a contestar al cuestionario ha sido de un $24 \%$ de las empresas contactadas.

Aunque el tamaño de la muestra es ligeramente menor que el planificado originalmente ${ }^{1}$, algunas de las características básicas de la población de empresas sobre las que se tenía información están proporcionalmente representadas en la muestra: tamaño, antigüedad, niveles de facturación, y distribución por sectores de actividad.

El cuestionario se refriere a las actividades cooperativas de innovación desarrolladas por las empresas. En concreto, se centra en la cooperación con los principales partners de la innovación y en la valoración que las Pymes hacen de ellos y del entorno regional. Las dimensiones que se recogen en el cuestionario son las siguientes:

1.- Actividad innovadora de las Pymes industriales guipuzcoanas.

2.- Cooperación en innovación y partners de cooperación de las Pymes industriales guipuzcoanas.

3.- Descripción de las relaciones de cooperación de las Pymes industriales guipuzcoanas.

4.- Valoración del entorno regional por parte de las Pymes industriales guipuzcoanas.

Hay que señalar que las características específicas de la cooperación se miden tomando como referencia un proyecto concreto de innovación/cooperación -el más relevante entre los abordados por la empresa durante los últimos tres años-, atendiendo tanto al agente (tipo, ubicación, valoración) como a la relación y al contenido y resultados de la cooperación.

\section{Perfil de la muestra}

El perfil de las empresas analizadas nos muestra una mayoría de empresas (60,6\%) de más de 20 años de antigüedad, no muy grandes (ni en número de empleados ni en facturación) y con especial peso en los sectores de metalurgia, artículos mecánicos y maquinaria.

\footnotetext{
${ }^{1}$ Desde un punto de vista estadístico, el tamaño de la muestra condiciona mucho la detección de significación estadística en las pautas de relación o diferencias que, sin embargo, pueden ser sustantivamente importantes. En consecuencia, las conclusiones del texto deben considerarse esencialmente descriptivas aunque algunas de las que así se destacan hubieran generado significación estadística si la muestra hubiera sido mayor. Hay que tener en cuenta que, trabajando con una población estadísticamente ya reducida (454) y metodológicamente difícil de encuestar (empresas), las posibilidades de conseguir una muestra amplia son sumamente pequeñas.
} 
Asimismo, hemos diferenciado a las empresas encuestadas a partir de la combinación de dos variables: a) si innova, esto es, si ha realizado alguna actividad innovadora en los últimos tres años; b) si coopera, esto es, si ha cooperado con algún agente externo en materia innovadora en los últimos tres años. De esta manera se han creado tres grupos: 1) Empresas que no innovan y no cooperan, 2) Empresas que innovan y no cooperan, 3) Empresas que innovan y cooperan.

Tabla 1.

Características básicas de la muestra de empresas ( $N=147)$.

\begin{tabular}{lcll}
\hline & $\%$ & & $\%$ \\
\hline Tamaño $\leq 50$ trabajadores & 62,6 & Facturación hasta 5 mill. euros & 46,3 \\
Tamaño > 50 trabajadores & 36,7 & Facturación 5 a 10 mill. euros & 26,8 \\
& & & \\
Sector Metalmecánica y bienes de equipo & 57,8 & & \\
Otros sectores & 42,2 & & \\
& 12,2 & & \\
Antigüedad $\leq 10$ años & 26,5 & NO INNOVA & 11,6 \\
Antigüedad 11 a 20 años & 29,9 & INNOVA PERO NO COOPERA & 25,2 \\
Antigüedad 21 a 30 años & 30,6 & INNOVA Y COOPERA & 63,3 \\
Antigüedad > 30 años & & & 147 \\
\hline Total N & 147 & Total N &
\end{tabular}

Fuente: Encuesta realizada a Pymes industriales 2008.

Como punto de partida, detectamos que la gran mayoría de las empresas encuestadas son empresas innovadoras: hasta el $88 \%$ afirmaron haber realizado actividades de innovación en los tres últimos años.

Respecto a las características objetivas disponibles de las empresas, no se han detectado pautas diferenciales relevantes en la presencia mayoritaria de actividad innovadora ni en función de la facturación, ni de la antigüedad, ni del sector; aunque para algunas combinaciones de características el porcentaje de empresas innovadoras supera el $90 \%$. 


\section{Resultados}

\section{Características de la cooperación con agentes externos de las Pymes guipuzcoanas}

Desde la teoría de sistemas de innovación se concibe la innovación como un proceso de aprendizaje interactivo que tiene lugar entre los agentes que componen la empresa, por un lado, y entre ésta y los agentes del entorno, por otro.

Los estudios de ámbito europeo que se centran en la influencia del entorno regional en la actividad innovadora de las Pymes destacan dos agentes del entorno que juegan un papel crucial en los procesos de innovación de estas empresas: clientes y proveedores. En este sentido, se resalta que un número limitado de relaciones usuario-proveedor duraderas y selectivas son normalmente las que moldean y restringen la mayoría de las actividades de innovación.

Agentes de cooperación para las Pymes

Para las Pymes guipuzcoanas entrevistadas es especialmente relevante la cooperación dentro de la cadena de valor: el $61,3 \%$ dice haber cooperado con clientes y el $53,8 \%$ con proveedores.

Asimismo, destaca el alto porcentaje de empresas que manifiesta colaborar con consultorías $(54,8 \%)$ y centros tecnológicos $(49,5 \%)$. En menor medida, las empresas cooperan con empresas de su propio grupo (38,7\%), universidades $(26,9 \%)$, centros de Formación Profesional (18,3\%), asociaciones empresariales (17,2\%), y clúster y Centros de Investigación Cooperativa (CIC) $(11,8 \%)$.

Tabla 2.

Partners para la cooperación en proyectos de innovación (\%) y valoraciones de la cooperación (Medias de valoración, escala de 1 a 5) ( $N=93$ ).

\begin{tabular}{lcc}
\hline & $\begin{array}{c}\text { \% empresas que } \\
\text { coopera }\end{array}$ & Valoración \\
\hline Clientes & 61,3 & 3,29 \\
Consultorias, ingenierías, empresas de servicios & 54,8 & 3,66 \\
Proveedores & 53,8 & 3,67 \\
Centros tecnológicos & 49,5 & 3,61 \\
Empresas de su mismo grupo & 38,7 & 3,81 \\
Universidades & 26,9 & 3,29 \\
Centros de Formación Profesional (FP) & 18,3 & 3,88 \\
Asociaciones empresariales sectoriales & 17,2 & 3,79 \\
Clusters; Centros de Investigación Cooperativa & 11,8 & 3,73 \\
\hline
\end{tabular}

Fuente: Encuesta realizada a Pymes industriales 2008. 
Preguntados por su valoración cuantitativa de la cooperación con cada tipo de agente, en general las valoraciones son claramente positivas (puntuaciones medias mayores que 3 en una escala 1-5), aunque la mayor frecuencia de colaboración con los agentes más mencionados aumenta también la variedad de respuesta en cuanto a valoración. Tomando como referencia un único proyecto de colaboración, el más importante de los últimos años, la preeminencia de la colaboración interempresarial se mantiene, ya que el $54,3 \%$ de las Pymes lo realizó dentro de la cadena de valor, mientras que el $45,3 \%$ fueron proyectos de colaboración con agentes de I+D, especialmente, con centros tecnológicos $(22,6 \%)$ o consultorías (14\%).

Por tanto, de los resultados de la encuesta se puede deducir una preferencia por parte de las empresas guipuzcoanas por cooperar con un agente empresarial frente a la cooperación con agentes de I+D (centros tecnológicos, universidades). Además, se pueden encontrar diferencias en las valoraciones de las experiencias de colaboración según esta clasificación de dos grandes tipos de partners.

$\mathrm{Si}$, en general, la satisfacción expresada con la experiencia de la cooperación es muy alta ( $75 \%$ expresan satisfacción clara, con una puntuación promedio de 3,87 en la escala 1-5), lo es más para las empresas que cooperaron con agentes empresariales (media 3,98 y $80 \%$ de alta satisfacción) que para las que lo hicieron con agentes I+D (media 3,74 y $69 \%$ de alta satisfacción).

Por otro lado, un $26,1 \%$ de las empresas que han colaborado con una empresa consideran que el impacto de la cooperación ha sido decisivo, siendo este porcentaje mayor que entre las empresas que han cooperado con un agente de $1+D(18,4 \%)$.

Tabla 3.

Impacto de la cooperación y esfuerzos destinados por la empresa según naturaleza del "partner" de cooperación ( $N=93)$.

\begin{tabular}{lccc}
\hline & Total & Empresa & Agente I+D \\
\hline $\begin{array}{l}\text { Satisfacción con cooperación (Escala 1-5) } \\
\begin{array}{l}\text { Impacto de la cooperación ha sido determinante } \\
\text { para éxito proyecto (\%) }\end{array}\end{array}$ & 3,87 & 3,98 & 3,73 \\
$\begin{array}{l}\text { Intensidad recursos económicos destinados al } \\
\text { proyecto. \% empresas que han sufragado más de }\end{array}$ & 22,4 & 26,1 & 18,4 \\
$\begin{array}{l}\text { la mitad del proyecto } \\
\text { Financiación del proyecto con recursos propios (\%) }\end{array}$ & 53,5 & 58,3 & \\
\hline
\end{tabular}

Fuente: Encuesta realizada a Pymes industriales 2008.

Otra característica diferencial de los proyectos de cooperación según el tipo de partner es la vía de financiación y el esfuerzo dedicado. Como veremos más adelante, la 
colaboración con agentes de I+D ocurre, principalmente, en el marco de programas de financiación pública, mientras que la cooperación entre y con empresas suele implicar mayor intensidad de recursos económicos propios dedicados, así como mayor implicación con el proyecto.

Esa imagen también se desprende en el comportamiento de las Pymes guipuzcoanas: hay una diferencia significativa en el porcentaje de empresas que declaran haber financiado su proyecto de colaboración más importante únicamente con recursos de la propia empresa cuando se trata de proyectos de colaboración con clientes, proveedores u otras empresas: el $67,4 \%$, frente a sólo el $35,7 \%$ de las empresas cooperando con agentes de I+D.

Además, se aprecia una mayor dedicación de recursos económicos por parte de la empresa en los proyectos con un agente empresarial frente a la cooperación efectuada con agentes de I+D. Más de la mitad de las empresas (58,7\%) que han cooperado con una empresa han sufragado más de la mitad del proyecto. Este porcentaje desciende al $47,5 \%$ en el caso de la cooperación con agentes de I+D. Esta mayor intensidad de recursos económicos destinados por la empresa al proyecto puede denotar un grado de implicación mayor en dicho proyecto.

\section{Reducida variedad de "partners" externos de cooperación}

Según la teoría de sistemas de innovación, los procesos de aprendizaje interactivo que establecen las empresas con agentes externos se basan, en gran medida, en relaciones de confianza necesarias para el intercambio de conocimiento tácito, no codificado. Adquieren relevancia en este contexto los "mercados organizados" (Lundvall 1992). Frente al carácter anónimo y con una función de mera transmisión de información codificada del mercado en la teoría neoclásica, estos mercados se caracterizan por el intercambio de información cualitativa, la cooperación y la confianza mutua. La aparición de estos elementos requiere la generación de relaciones selectivas de carácter estable y duradero entre los actores.

De nuestra encuesta se puede deducir que la creación y transferencia de conocimiento necesario para la innovación se produce a partir del establecimiento por parte de las Pymes de un conjunto reducido de relaciones a largo plazo con agentes externos basadas en normas recíprocas de cooperación y confianza mutua. Ello se puede apreciar en tres rasgos de las relaciones de cooperación que establecen las Pymes guipuzcoanas en sus proyectos de innovación: reducida variedad de partners de cooperación, regularidad de la cooperación e importancia de la confianza.

Respecto al primero de dichos rasgos, se ha calculado el número de agentes diferentes con los que cada pyme entrevistada ha manifestado cooperar en proyectos de innovación durante los últimos tres años. Con relación a la clasificación manejada de 9 tipos de agentes, hay una considerable variedad de posiciones de las empresas en cuanto a la extensión de sus redes de colaboración, pero cabe destacar que más de la mitad de las empresas sólo ha colaborado como máximo con tres tipos diferentes. 
Además, hay que destacar que las empresas con más de 50 empleados presentan una mayor variedad de relaciones externas que las Pymes más pequeñas.

Tabla 4.

Variedad de agentes con los que se ha cooperado

según tamaño de la empresa ( $N=93)$.

\begin{tabular}{lccc}
\hline Variedad Partners de cooperación & Total & $\leq 50$ empleados & $>50$ empleados \\
\hline 1 único tipo de partner & 22,6 & 23,6 & 21,1 \\
2 partners diferentes & 14,0 & 16,4 & 10,5 \\
3 partners diferentes & 19,4 & 20,0 & 18,4 \\
4 partners diferentes & 18,3 & 16,4 & 21,1 \\
5 partners diferentes & 11,8 & 10,9 & 13,2 \\
$>5$ partners diferentes & 13,9 & 12,7 & 15,7 \\
Total & 100,0 & 100,0 & 100,0 \\
\hline
\end{tabular}

Fuente: Encuesta realizada a Pymes industriales 2008.

No se puede concluir que el número de colaboradores externos distintos con que se han realizado proyectos de cooperación en algún momento tenga que ver con la antigüedad o el sector. La casuística de las empresas es simplemente diversa.

Regularidad de la cooperación con agentes externos

Estas relaciones son además duraderas en el tiempo, como se puede deducir de las respuestas de las empresas a la pregunta sobre la regularidad de la cooperación. Así, un $43,3 \%$ de las empresas manifiesta haber cooperado más de tres veces con el mismo agente con el que desarrolló últimamente un proyecto de innovación relevante, siendo un $21,1 \%$, las empresas que han cooperado 203 veces con el mismo agente y un $35,6 \%$ las que lo han hecho por primera vez.

En cierto sentido, las relaciones de cooperación con agentes de I+D tienden a ser especialmente estables y duraderas. En general, cabría afirmar que una vez que se ha tenido una experiencia de cooperación positiva con un agente de $++D$, se tiende a repetir la colaboración en ulteriores proyectos, destacando específicamente la cooperación con la red de centros tecnológicos, que es la que alcanza mayores índices de regularidad, a pesar de no ser la más común entre las Pymes guipuzcoanas. En el caso de cooperación entre empresas, las relaciones están más polarizadas: hay un importante porcentaje de relaciones de largo recorrido $(45,8 \%)$ frente a un importante número de casos en los que se ha cooperado por primera vez $(41,7 \%)$. 
Tabla 5.

Regularidad de cooperación con el partner que se ha colaborado en el proyecto de innovación según naturaleza del "partner" ( $N=93)$.

\begin{tabular}{lccc} 
Regularidad de la cooperación (\%) & Total & Empresa & Agente I+D \\
\hline Por primera vez & 35,6 & 41,7 & 28,6 \\
Dos o tres veces & 21,1 & 12,5 & 31,0 \\
Más de tres veces & 43,3 & 45,8 & 40,5 \\
Total & 100,0 & 100,0 & 100,0 \\
\hline
\end{tabular}

Fuente: Encuesta realizada a Pymes industriales 2008.

Entre los agentes de cooperación más frecuentes, los centros tecnológicos alcanzan también los índices máximos de regularidad: más de la mitad $(52,4 \%)$ de las empresas con proyectos de cooperación con este tipo de agente declaran una trayectoria de innovación con el mismo.

También es destacable que ninguna característica "objetiva" del tipo de proyectos (fase, contenidos, esfuerzo de la empresa, financiación) ni del tipo de empresa (tamaño, sector, antigüedad) marca diferencias en la regularidad de la cooperación con un mismo agente, por lo que efectivamente los resultados apuntan a la importancia de elementos intangibles (como la confianza) en el establecimiento de relaciones duraderas de cooperación con un agente externo.

Relevancia de la confianza

La confianza juega un papel importante en el establecimiento de relaciones de cooperación por parte de las empresas. Al subrayar las dificultades que se han encontrado en la cooperación con el agente externo en el proyecto de innovación, el elemento menos percibido como obstáculo ha sido precisamente la falta de confianza. No llegan a un 13\% las empresas con proyectos de cooperación que muestran alguna preocupación por este elemento. Además, este obstáculo tiene significativamente aún menos importancia en el caso de la cooperación con un agente de $\mathrm{I+D}$, precisamente donde la regularidad de la cooperación es mayor. Se puede decir que la cooperación reiterada a lo largo del tiempo da lugar a que se establezcan relaciones basadas en la confianza. 
Tabla 6.

Importancia de los diferentes obstáculos en la cooperación con un agente externo en el proyecto de innovación según naturaleza del "partner" (N=93).

\begin{tabular}{lccr}
\hline Medias de valoración (escala 1 a 5) & Total & Empresa & Agente I+D \\
\hline Falta de confianza & 1,76 & 1,98 & 1,50 \\
Cultura poco favorable a la cooperación & 1,94 & 2,09 & 1,78 \\
Rigideces organizativas & 2,23 & 2,41 & 2,02 \\
Falta de conocimientos en nuestra propia organización & 2,29 & 2,35 & 2,21 \\
Falta de conocimientos del agente con el que ha cooperado & 1,93 & 2,00 & 1,85 \\
Falta de recursos económicos & 2,45 & 2,38 & 2,54 \\
Falta de tiempo para el proyecto & 2,81 & 2,92 & 2,69 \\
Falta de capacitación para gestionar proyectos de & & & \\
innovación en cooperación & 2,16 & 2,34 & 1,95 \\
Dificultades de comunicación & 1,97 & 2,21 & 1,69 \\
\hline
\end{tabular}

Fuente: Encuesta realizada a Pymes industriales 2008.

Se detecta además una coherencia en la valoración que las empresas realizan de las dificultades más intangibles, ya que se produce una considerable concordancia de respuestas (correlaciones mayores de 0,50 ) en la percepción de las dificultades de falta de confianza, problemas de comunicación y cultura poco favorable a la cooperación: las empresas que menos importancia dan a uno de estos aspectos, tampoco se la dan a los otros, y las que más importancia les otorgan, les dan más a todos ellos.

\section{Influencia del sistema regional de innovación en la actividad innovadora de las Pymes}

Según la teoría de sistemas de innovación regional estas relaciones de cooperación en innovación, caracterizadas por la confianza y el intercambio de conocimiento tácito, se basan, en gran medida, en la proximidad geográfica. Además, de acuerdo a los estudios realizados bajo esta perspectiva, la existencia de un entorno regional que favorezca la actividad innovadora es especialmente importante en el caso de las Pymes, ya que éstas se encuentran más imbricadas en el entorno regional que las empresas más grandes.

Ello queda corroborado por los resultados de nuestra encuesta. Un $48 \%$ de las relaciones de cooperación de las empresas entrevistadas se dan con agentes ubicados en el territorio histórico de Gipúzcoa. El porcentaje aumenta hasta el 68\% si consideramos las relaciones dentro de la región de la Comunidad Autónoma del País Vasco (CAPV).

La proximidad geográfica es especialmente importante en la cooperación con agentes de I+D. Un $90 \%$ de las empresas que cooperan con agentes de I+D lo hacen con un agente ubicado en la provincia o región, frente al 50\% de las empresas que colaboran con otra empresa ubicada en la región. 
Tabla 7.

Ubicación del "partner" de cooperación según su naturaleza (N=93).

\begin{tabular}{lccc}
\hline Ubicación del partner (\%) & Total & Empresa & Agente 1+D \\
\hline Provincia & 48,9 & 40,0 & 59,5 \\
Región & 19,6 & 10,0 & 31,0 \\
Estado & 17,4 & 26,0 & 7,1 \\
Unión Europea (UE) & 12,0 & 20,0 & 2,4 \\
Fuera de la Unión Europea (UE) & 2,2 & 4,0 & 0,0 \\
Total & 100,0 & 100,0 & 100,0 \\
\hline
\end{tabular}

Fuente: Encuesta realizada a Pymes industriales 2008.

Uno de los elementos más estudiados sobre la influencia que ejerce el entorno regional en la actividad innovadora de las Pymes es la relación que éstas mantienen con las infraestructuras de $I+D$, creadas con el objeto de que actúen como soporte a las actividades de innovación de las Pymes.

Precisamente, uno de los rasgos del sistema regional de innovación en la CAPV es la existencia de una importante red de centros tecnológicos dedicada, principalmente, a la investigación aplicada y a la transferencia tecnológica a las Pymes del entorno, y gran parte de los cuales se encuentran ubicados en Guipúzcoa. El relevante papel de estos agentes en los procesos de innovación de las Pymes se manifiesta, como destacamos al principio, en el alto porcentaje de empresas encuestadas (49,5\%) que ha cooperado con estos agentes específicos en el marco de proyectos de innovación, aún cuando en conjunto las preferencias de colaboración se inclinan más por el entorno empresarial.

Según los resultados de nuestra encuesta, las fuentes institucionales de información tienen significativamente mayor relevancia cuando los proyectos de cooperación se realizan con agentes de I+D. Además, las innovaciones de producto generan mayor cooperación con agentes de I+D $(61,9 \%)$ frente a las de procesos. Por otro lado, la cooperación con los agentes de I+D se produce sobre todo en la fase de diseño (42,9\%), frente a la cooperación con otras empresas que surge principalmente en la fase de desarrollo $(41,2 \%)$.

En el caso de la cooperación entre empresas, hay más variedad de formatos, aunque el tipo concreto de colaboración se centra en la realización de proyectos conjuntos de innovación $(42,2 \%)$ y secundariamente en el intercambio organizado de información y experiencias $(37,5 \%)$. Para las cooperaciones con agentes de I+D, la subcontratación de servicios $(42,2 \%)$ es la forma de colaboración más frecuente, aunque el porcentaje de proyectos conjuntos entre las cooperaciones es casi equivalente $(40 \%)$. 
Tabla 8.

Fuentes de información y contenido de la cooperación según naturaleza del agente ( $N=93)$.

\begin{tabular}{|c|c|c|c|}
\hline & Total & Empresa & Agente I+D \\
\hline \multicolumn{4}{|c|}{$\begin{array}{l}\text { Fuentes de información para la cooperación } \\
\text { con dicho agente }(\%)\end{array}$} \\
\hline Fuentes del mercado & 41,3 & 48,0 & 33,3 \\
\hline Fuentes institucionales & 10,9 & 2,0 & 21,4 \\
\hline \multicolumn{4}{|c|}{ Contenido de la innovación (\%) } \\
\hline Producto & 53,8 & 47,1 & 61,9 \\
\hline Proceso & 39,8 & 49,0 & 28,6 \\
\hline \multicolumn{4}{|c|}{ Fase del proyecto en que se ha cooperado (\%) } \\
\hline Diseño & 37,6 & 33,3 & 42,9 \\
\hline Desarrollo & 37,6 & 41,2 & 33,3 \\
\hline
\end{tabular}

Fuente: Encuesta realizada a Pymes industriales 2008.

Otro aspecto en el que el sistema regional de innovación favorece la innovación en Pymes es a través de la puesta en marcha de instrumentos de financiación de sus actividades de innovación. Hay que señalar que un $43,2 \%$ de las empresas encuestadas ha contado con financiación pública para la realización del proyecto de innovación más importante llevado a cabo en los últimos tres años. La financiación pública beneficia en mayor medida a las Pymes que colaboran con agentes de I+D y, como veremos en el siguiente apartado, a las Pymes más grandes. Un $63,2 \%$ de las cooperación con agentes de I+D han recibido financiación pública; en el caso de cooperación con empresas este porcentaje se reduce al $25,6 \%$.

Tabla 9.

Obtención de financiación pública para el proyecto de innovación según partner de la cooperación ( $N=93)$.

\begin{tabular}{lccc}
\hline Porcentaje de financiación pública (\% empresas) & Total & Empresa & Agente I+D \\
\hline Menos del 10\% & 0,0 & 0,0 & 0,0 \\
Entre el 10\% y el 24\% & 16,0 & 11,6 & 21,1 \\
Entre el 25\% y el 49\% & 13,6 & 9,3 & 18,4 \\
El 50\% o más & 13,6 & 4,7 & 23,7 \\
Sin financiación pública & 56,8 & 74,4 & 36,8 \\
Total & 100,0 & 100,0 & 100,0 \\
\hline
\end{tabular}

Fuente: Encuesta realizada a Pymes industriales 2008. 
El entorno regional es, pues, clave en los procesos de innovación de las Pymes guipuzcoanas que circunscriben en gran medida, como acabamos de ver, sus relaciones externas al nivel regional. Como se puede ver en la siguiente tabla, las valoraciones que realizan las Pymes encuestadas de todos los aspectos propuestos sobre el sistema regional de innovación resultan por término medio positivas (medias de alrededor de 3 en escala 1-5), aunque hay diferencias de matiz según aspectos, si medimos el porcentaje de valoraciones extremas en la escala. Los elementos mejor valorados son los centros tecnológicos $(3,25)$, seguido del acceso a proveedores $(3,18)$, las infraestructuras físicas $(3,06)$ y los centros de FP $(3,02)$.

Tabla 10.

Valoración del entorno regional CAPV (N=147).

\begin{tabular}{lcccc}
\hline Valoración 1-5 & $\begin{array}{c}\text { Media } \\
\text { Total }\end{array}$ & $\begin{array}{c}\text { \% valora peor } \\
(1-2)\end{array}$ & $\begin{array}{c}\text { \% valora mejor } \\
(4-5)\end{array}$ & $\begin{array}{c}\text { Media no } \\
\text { cooperan (N=53) }\end{array}$ \\
\hline Red centros tecnológicos & 3,25 & 22,1 & 35,9 & 3,04 \\
Acceso a proveedores & 3,18 & 23,2 & 42,7 & 3,14 \\
Infraestructuras físicas & 3,07 & 26,2 & 30,0 & 3,00 \\
Centros de FP & 3,03 & 35,2 & 39,3 & 3,12 \\
Acceso a clientes & 2,99 & 28,9 & 30,4 & 2,94 \\
Disponibilidad mano obra cualificada & 2,93 & 37,1 & 31,4 & 2,98 \\
Universidades & 2,92 & 36,3 & 31,4 & 2,76 \\
Acceso a programas y ayudas públicas & 2,90 & 38,8 & 37,3 & 2,76
\end{tabular}

Fuente: Encuesta realizada a Pymes industriales 2008.

En general, las Pymes con experiencia reciente en proyectos de cooperación tienden a valorar algo mejor el entorno que las empresas que no cooperan con otros agentes, aunque éstas no valoran de manera negativa el entorno.

Sin embargo, entre las empresas que sí cooperan, algunas valoraciones del entorno regional son significativamente diferentes en función de su capacidad de captar financiación pública para proyectos de cooperación. Así, por ejemplo, las valoraciones medias son significativamente mejores para las Pymes que desarrollaron sus proyectos importantes contando con financiación pública en aspectos como: "acceso a financiación", "infraestructuras físicas del sistema", "red de centros tecnológicos" e incluso "acceso a clientes".

\section{Influencia del tamaño y la capacidad de absorción en la utilización de los recursos del sistema regional}

Además de subrayar los efectos que puede tener el entorno en la actividad innovadora de las Pymes, diversos estudios realizados bajo el prisma del sistema de innovación resaltan la importancia de las bases internas de conocimiento en los procesos de innovación de 
las Pymes. En este contexto se subraya la importancia de que las empresas hayan desarrollado competencias internas como condición indispensable para que puedan establecer relaciones fructiferas de cooperación con agentes externos y sean capaces de hacer uso de información externa e integrarla con la procedente del interior de la empresa. En este apartado se atiende al concepto de capacidad de absorción y cómo la capacidad para captar, evaluar y utilizar nuevo conocimiento en gran medida es función del nivel de conocimiento acumulado.

De los resultados de nuestra encuesta se deduce que, entre las empresas que no han desarrollado recientemente proyectos de cooperación, algunos de los motivos más aducidos hacen referencia a ciertos aspectos de esa capacidad interna: coste económico, renuencia a asumir riesgo, dificultad para encontrar partners para la cooperación en el entorno o falta de cultura para la cooperación.

Tabla 11.

Valoración de motivos para la no cooperación ( $N=54)$.

\begin{tabular}{lcc}
\hline Importancia 1-5 & Media & $\begin{array}{c}\text { \% mayor importancia } \\
(4-5)\end{array}$ \\
\hline Coste económico & 3,13 & 42,5 \\
No existe cultura cooperación & 3,00 & 33,4 \\
Agentes de su entorno no añaden valor a sus proyectos de innovación & 2,95 & 39,0 \\
Dificultad para encontrar partners & 2,95 & 33,3 \\
Riesgo inasumible & 2,91 & 41,9 \\
Su mercado no es sensible a innovaciones & 2,60 & 29,7 \\
Malas experiencias & 2,48 & 33,3 \\
Peligro confidencialidad & 2,46 & 20,8 \\
\hline
\end{tabular}

Fuente: Encuesta realizada a Pymes industriales 2008.

Entre las empresas que sí han tenido proyectos de cooperación, también algunos de los obstáculos principales señalados (tabla 6) además de la falta de tiempo y recursos económicos, aluden a capacidades internas de la empresa como "falta de conocimientos en la propia organización" o "rigideces organizativas". Si bien en el caso de las empresas que cooperan con otros agentes todos los tipos de dificultades son menos determinantes (valoraciones medias más bajas), comparativamente las mencionadas son más importantes, como hemos visto anteriormente.

Dentro de la encuesta las variables que reflejan en mayor medida la capacidad interna de la empresa son el tamaño y la existencia de oficina técnica y/o departamento de I+D.

La variable "tamaño" muestra una relación importante con muchos de los aspectos de las actividades de innovación y, por supuesto, de cooperación. El tamaño es una 
variable que influye en las características medidas de la actividad innovadora de las Pymes, en las relaciones de cooperación que establecen en dichos procesos, así como en la relación con el entorno regional para su actividad innovadora.

Como mínimo, el desarrollo de actividad innovadora es más palpable cuanto mayor es el tamaño de la empresa (tabla 12). Además, la formalización de la innovación está claramente relacionada con el tamaño empresarial. La presencia de oficina técnica y/o departamento de I+D es más frecuente entre las Pymes de más de 50 empleados.

Hay que destacar que la realización de actividades de innovación de las Pymes se canaliza en gran medida a través de la oficina técnica. La existencia de un departamento de I+D es menos frecuente y su existencia se circunscribe a las Pymes más grandes. Un $64,8 \%$ de las empresas con más de 50 empleados entrevistados cuentan con este tipo de estructura, frente al 30,8 \% entre las empresas de menor tamaño. Así las cosas, se deduce que las oficina técnicas de las Pymes encuestadas compatibilizan las actividades del día a día (estudio de pedidos, presupuestación, desarrollo de máquinas, adaptación de procesos productivos) con la realización de actividades a medio-largo plazo como vigilancia tecnológica y asistencia a ferias, etc.

Por otro lado, las relaciones de cooperación para la innovación son tanto más frecuentes cuanto mayor es el tamaño de las empresas: un $70,4 \%$ de las Pymes con más de 50 empleados coopera con agentes externos en sus procesos de innovación, descendiendo este porcentaje al 60\% en las Pymes de menos de 50 empleados.

Tabla 12.

Intensidad de innovación y cooperación y estructura para la innovación en cooperación según tamaño.

\begin{tabular}{|c|c|c|}
\hline Total empresas $(\mathrm{N}=147)$ & $\leq 50$ empleados & $>50$ empleados \\
\hline Empresas que innovan (\%) & 85,7 & 92,6 \\
\hline Existencia oficina técnica (\%) & 76,9 & 79,6 \\
\hline Existencia departamento I+D (\%) & 30,8 & 64,8 \\
\hline Cooperación en innovación (\%) & 60,4 & 70,4 \\
\hline \multicolumn{3}{|l|}{ Importancia del factor "Necesidades cubiertas" } \\
\hline para la no cooperación (Media valoración 1 a 5) & 3,04 & 2,73 \\
\hline \multicolumn{3}{|l|}{ Empresas que han cooperado ( $\mathrm{N}=93$ ) } \\
\hline $\begin{array}{l}\text { La propia empresa (\%) } \\
\text { Recursos económicos }(\% \text { empresas que han financiado }\end{array}$ & 44,4 & 37,8 \\
\hline $\begin{array}{l}\text { más de la mitad del proyecto) } \\
\text { Recursos humanos (\% empresas que destinan una persona }\end{array}$ & 41,5 & 72,7 \\
\hline Equivalencia a Dedicación Plena (EDP) o más al proyecto) & 48,9 & 60,5 \\
\hline \% Empresas que han obtenido financiación pública & 34,0 & 58,1 \\
\hline Satisfacción cooperación (Media valoración 1 a 5) & 3,75 & 4,05 \\
\hline
\end{tabular}

Fuente: Encuesta realizada a Pymes industriales 2008. 
De nuestro estudio se deriva que la existencia de algún tipo de estructura para la innovación favorece de manera significativa el establecimiento de relaciones con otros agentes externos. Las empresas que disponen de una estructura para la actividad innovadora cooperan significativamente en mayor medida con agentes externos en materia de innovación. Un $72,8 \%$ de las empresas que cuentan con oficina técnica y/o departamento de I+D cooperan con agentes externos en I+D+I mientras que entre las empresas que no disponen de alguna estructura para estas actividades, la gran mayoría no coopera con agentes externos.

Para la información sobre socios de cooperación en materia de innovación, en las empresas más pequeñas tienen más peso las fuentes internas $(44,4 \%)$ que en el caso de las Pymes de mayor tamaño (37,8\%). Ello puede denotar una falta de capacidades para la búsqueda y selección de información relevante del exterior en el caso de las empresas más pequeñas.

Se detecta que las Pymes de mayor tamaño destinan mayores recursos económicos y humanos a la realización de proyectos de innovación. Asimismo, se puede deducir que las Pymes más grandes aprovechan de mejor manera los resultados de la cooperación ya que manifiestan unos mayores niveles de satisfacción (media 4,05 ) con los resultados de dicha cooperación que las Pymes de menos de 50 empleados $(3,75)$.

Los agentes externos que inciden en los procesos de innovación de las Pymes encuestadas varían según el tamaño. Ya hemos visto al principio que la variedad de agentes externos con los que cooperan las Pymes aumenta con el tamaño. También hemos podido apreciar que los centros de FP son mejor valorados por las empresas más pequeñas mientras que las empresas más grandes valoran mejor a los centros tecnológicos.

Se deduce de nuestra encuesta que estos centros alcanzan en mayor medida a las Pymes más grandes. Así las cosas, las Pymes de más de 50 empleados presentan mayores niveles de colaboración con estos agentes, un $65,8 \%$, frente al $38,2 \%$ de las Pymes de menos de 50 empleados que manifiestan haber colaborado con centros tecnológicos. Hay que tener en cuenta que normalmente estas Pymes más grandes disponen de centros tecnológicos dentro de su grupo empresarial o forman parte de los patronatos de estos centros. Las Pymes más pequeñas no tienen tradición de colaboración con estos agentes y la relación con ellos se limita a la prestación de servicios puntuales.

Entre las razones aducidas para la no cooperación llama la atención que las empresas más pequeñas manifiesten que tienen cubiertas sus necesidades tecnológicas, siendo las Pymes más grandes las que hacen referencia al coste económico y a la falta de información. Precisamente, uno de los problemas de la transferencia de conocimiento y tecnología por parte de agentes de I+D a las Pymes más pequeñas, es la dificultad de éstas para articular sus necesidades en materia de innovación y el hecho de que en ocasiones no son conscientes de ellas.

Además de la percepción diferencial de los centros tecnológicos como partner y como elemento singular del sistema de I+D+l, también destaca el hecho de que las 
Pymes más grandes valoran de manera significativamente más positiva el resto de infraestructuras de I+D.

Tabla 13.

Valoración de los "partners" de cooperación según tamaño (N=93).

\begin{tabular}{|c|c|c|c|}
\hline Medias de valoración (escala 1 a 5) & Total & $\leq 50$ empleados & $>50$ empleados \\
\hline Empresas de su mismo grupo $(\mathrm{N}=36)$ & 3,81 & 3,72 & 3,89 \\
\hline Proveedores de equipos, material, componentes o software $(\mathrm{N}=48)$ & 3,67 & 3,57 & 3,80 \\
\hline Clientes $(N=56)$ & 3,64 & 3,62 & 3,68 \\
\hline Consultorías, ingenierías y/u otras empresas de servicios ( $N=50)$ & 3,66 & 3,72 & 3,57 \\
\hline Universidades $(\mathrm{N}=24)$ & 3,29 & 3,38 & 3,18 \\
\hline Centros tecnológicos ( $\mathrm{N}=46)$ & 3,61 & 3,48 & 3,72 \\
\hline Centros de FP $(\mathrm{N}=17)$ & 3,88 & 3,92 & 3,75 \\
\hline Clusters y/o centros de investigación cooperativa $(\mathrm{CIC})(\mathrm{N}=11)$ & 3,73 & 3,60 & 3,83 \\
\hline Asociaciones empresariales sectoriales $(\mathrm{N}=14)$ & 3,79 & 3,56 & 4,20 \\
\hline
\end{tabular}

Fuente: Encuesta realizada a Pymes industriales 2008.

En las valoraciones del entorno (tabla 14), las Pymes de mayor tamaño valoran de manera más positiva elementos como la disponibilidad de mano de obra cualificada, la red de centros tecnológicos, las universidades, el acceso a programas de financiación pública y las infraestructuras físicas. Por otro lado las Pymes de menor tamaño (con menos de 50 empleados) presentan una valoración algo más alta del acceso en la región a empresas dentro de la cadena de valor (clientes y proveedores), así como de los centros de FP. En este último caso, hay que señalar que se produce un aumento significativo de la valoración de este agente a medida que disminuye el tamaño de la empresa. Este resultado apunta la importancia que los centros de formación profesional pueden tener en los procesos de innovación de las Pymes, sobre todo de las de menor tamaño. La potencialidad de este agente no ha sido todavía suficientemente reconocida por parte de las políticas de I+D.

Tabla 14.

Valoración del entorno regional según tamaño ( $N=147)$.

\begin{tabular}{lccc}
\hline Medias de valoración (escala 1 a 5) & Total & $\leq 50$ empleados & $>50$ empleados \\
\hline Disponibilidad mano obra cualificada & 2,93 & 2,81 & 3,14 \\
Acceso a clientes & 2,99 & 2,99 & 2,98 \\
Acceso a proveedores & 3,18 & 3,23 & 3,10 \\
Red centros tecnológicos & 3,25 & 3,14 & 3,42 \\
Universidades & 2,92 & 2,81 & 3,11 \\
Centros de Formación Profesional (FP) & 3,03 & 3,16 & 2,78 \\
Acceso a programas y ayudas públicas & 2,90 & 2,80 & 3,06 \\
Infraestructuras físicas & 3,07 & 2,99 & 3,22 \\
\hline
\end{tabular}

Fuente: Encuesta realizada a Pymes industriales 2008. 
Por otro lado, las Pymes más grandes acceden en mayor medida a programas de financiación pública. Un 37,7\% de las Pymes más pequeñas han contado con ayuda pública para la relación del proyecto de innovación más importante de los últimos tres años, frente al $62,9 \%$ de las Pymes de más de 50 empleados que han contado con estas ayudas. En este sentido, se aprecia la necesidad de contar con una serie de capacidades para poder gestionar los proyectos realizados en el marco de estas ayudas. Además, la colaboración con centros tecnológicos incide de manera positiva en la obtención de financiación pública. La colaboración con estos agentes se realiza, principalmente, en el marco de programas públicos de ayuda a la I+D e innovación que impulsan la colaboración con estos agentes, además los centros tecnológicos prestan un apoyo indispensable para la gestión de la documentación de estos proyectos.

Tabla 15.

Obtención de financiación pública para proyecto de innovación según tamaño (N=93).

\begin{tabular}{|c|c|c|c|}
\hline Fuentes de financiación del proyecto (\% empresas) & Total & $\leq 50$ empleados & $>50$ empleados \\
\hline $\begin{array}{l}\text { Exclusivamente con recursos de la empresa } \\
\text { Cofinanciado con recursos provenientes de }\end{array}$ & 52,3 & 62,3 & 37,1 \\
\hline $\begin{array}{l}\text { convocatorias para } \\
\text { Exclusivamente con recursos provenientes de }\end{array}$ & 45,5 & 35,8 & 60,0 \\
\hline $\begin{array}{l}\text { convocatorias públicas } \\
\text { Total }\end{array}$ & $\begin{array}{r}2,3 \\
100,0\end{array}$ & $\begin{array}{r}1,9 \\
100,0\end{array}$ & $\begin{array}{r}2,9 \\
100,0\end{array}$ \\
\hline
\end{tabular}

Fuente: Encuesta realizada a Pymes industriales 2008.

\section{Conclusiones}

La teoría de los sistemas de innovación muestra que las innovaciones se producen, principalmente, a través de un proceso de aprendizaje interactivo dentro de la empresa, y entre la empresa y otras organizaciones. Esta interacción tiene lugar dentro de un entorno institucional y cultural.

El modelo interactivo de innovación es especialmente relevante en sectores enfocados a la realización de innovaciones incrementales y en los que se coopera con un conjunto reducido de actores (principalmente clientes y proveedores) en relaciones caracterizadas por la confianza, el intercambio de información cualitativa y la cooperación (aunque no exentas de jerarquía o diferenciales de poder de negociación entre los agentes).

De los resultados de este trabajo se deriva que la cooperación en innovación de las Pymes industriales guipuzcoanas se ajusta en gran medida a este modelo de innovación. 
Las relaciones con agentes externos en proyectos de innovación de estas empresas se materializan en cooperaciones con una reducida variedad de agentes, a largo plazo y basadas en la confianza. Asimismo, se caracterizan por su regularidad y estabilidad.

Este modelo interactivo de innovación se produce preferentemente dentro de un "sistema territorialmente imbricado", donde la proximidad geográfica, social y cultural entre los actores juega un papel crucial en los procesos de cooperación en innovación. Uno de los principales resultados de esta investigación es que las relaciones de cooperación se encuentran altamente localizadas dentro del territorio histórico (Gipúzcoa) y la región (CAPV).

En línea con los resultados de los estudios realizados bajo el prisma de los sistemas regionales de innovación a nivel internacional, los agentes dentro de la cadena de valor (clientes y proveedores) son especialmente relevantes para las Pymes encuestadas como partners para la cooperación. Asimismo, se manifiesta una preferencia por parte de las empresas guipuzcoanas por cooperar con un agente empresarial frente a la cooperación con agentes de I+D (centros tecnológicos, universidades). Las Pymes que han colaborado con un agente empresarial, en comparación con las que han cooperado con un agente de I+D, están más satisfechas de la cooperación y consideran que el impacto de dicha cooperación es más decisivo para el éxito del proyecto. Además, las Pymes que colaboran con otras empresas manifiestan una mayor implicación en el proyecto (medido por la intensidad de los recursos económicos dedicados).

No obstante, frente a los resultados de algunos estudios internacionales, en el caso de las Pymes industriales guipuzcoanas se detecta un mayor peso relativo de la cooperación con agentes de I+D (concretamente con la infraestructura de centros tecnológicos) en los procesos de innovación. De esta manera, destaca el alto porcentaje de empresas que manifiesta haber colaborado con este agente. Además las relaciones de cooperación con estos agentes permiten a las empresas beneficiarse de programas públicos de ayuda a la I+D e innovación.

El tamaño y la capacidad interna para la realización de actividades innovadoras influyen en gran medida en los patrones de cooperación de las Pymes industriales guipuzcoanas. Así, las relaciones de cooperación para la innovación son tanto más frecuentes cuanto mayor es el tamaño de las empresas o cuando existe una estructura para la realización de actividades de I+D e innovación (oficina técnica/departamento I+D). Además, las Pymes de mayor tamaño destinan mayores recursos económicos y humanos a la realización de proyectos de innovación. La variedad de agentes externos con los que cooperan las Pymes aumenta también con el tamaño, así como el nivel de cooperación con los centros tecnológicos: son las Pymes más grandes las que se benefician en mayor medida de la cooperación con estos agentes. Por otro lado, las Pymes más pequeñas valoran de manera especialmente positiva los centros de FP. A nuestro juicio, dada la importancia de este agente para las Pymes más pequeñas, con menores capacidades en innovación, debería ser más tenido en cuenta por las políticas de I+D.

En definitiva, en casos como el analizado, cabe afirmar que el sistema de innovación regional facilita los procesos de innovación desarrollados en las Pymes industriales. 


\section{REFERENCIAS BibLIOGRÁFICAS}

Asheim B. T. and L. Coenen. 2005. "Knowledge Bases and Regional Innovation Systems: Comparing Nordic Clusters." Research Policy 34:1173-1190.

Asheim B. y M. Gertler. 2005. "The Geography of Innovation: Regional Innovation Systems". Pp. 291317 en The Oxford Handbook of Innovation compilado por by J. Fagerberg, D. Mowery and R. Nelson. Oxford: Oxford University Press.

Braczyk, H., P. Cooke y R. Heidenreich, eds. 1996. Regional Innovation Systems. The Role of Governances in a Globalized World. Londres: University College London Press.

Breschi, S. y F. Malerba. 1997. "Sectoral innovation systems: Technological regimes, shumpeterian dynamics, and spatial boundaries". Pp. 130-156 en Systems of Innovation: Technologies, Institutions and Organisations, edited by C. Edquist. London: Pinter.

Cooke, P., M. Gómez y G. Etxebarría. 1997. "Regional Innovation Systems: Institutional and Organizational Dimensions", Research Policy 26:475-491.

Cooke, P. y K. Morgan. 1998. The Associational Economy: Firms, Regions and Innovation. Oxford: Oxford University Press.

Copus, A., D. Skuras. y K. Tsegenidi. 2008. "Innovation and peripherality: an empirical comparative study of SMEs in six european union member countries." Economic Geography 84:51-82.

Doloreux, D. 2003. "Regional innovation systems in the periphery: the case of Beauce in Quebec (Canada)." International Journal of innovation management 7:67-94.

Doloreux, D. 2004. "Regional innovation systems in Canada: a comparative study." Regional Studies 38:479-492.

Edquist, C. 1997. Systems of Innovation: Technologies, Institutions, and Organizations. Londres: Pinter.

Freel, M. S. 2000. "Strategy and structure in innovative manufacturing SMEs: the case of an English Region." Small Business Economics 15:27-45.

Freel, M. S. 2003. "Sectoral patterns of small firm innovation, networking and proximity." Research Policy 32:751-770.

Freel, M. S. y R. T. Harrison. 2006. "Innovation and cooperation in the small firm sector: Evidence from 'Northern Britain'." Regional Studies 40:289-305.

Fritsch, M. 2001. "Co-operation in regional innovation systems." Regional Studies 35:297-307.

Gebauer, A., C. Woon Nam y R. Parsche. 2005. "Regional technology policy and factors shaping local innovation networks in small German cities." European Planning Studies 13:661-683.

Grotz, R. y B. Braun. 1997. "Territorial or transnational networking: spatial aspects of technology oriented cooperation within the German mechanical Engineering Industry." Regional Studies 31:545-557. 
Hassink, R. 1997. "Technology transfer infrastructures: some lessons from experiences in Europe, the US and Japan." European Planning Studies 5:351-370.

Johnson, B. 1992. "Institutional learning". Pp. 23-44 en National Systems of Innovation, edited by B. Lundvall. Londres: Pinter.

Kaufmann, A. y F. Tödtling. 2000. "Systems of innovation in traditional industrial regions: the case of Styria in a comparative perspective." Regional Studies 34:29-40.

Kaufmann, A. y F. Tödtling. 2002. "How effective is innovation support for SMEs? An analysis of the region of Upper Austria." Technovation 22:147-159.

Kaufmann, A. y F. Tödtling. 2003. "Innovation pattern of SMEs". Pp. 78-115 en B. Asheim, A. Isaksen, C. Nauwelaers y F. Tödtling (eds.) Regional innovation policy for small-medium enterprises. Cheltenham: Edwar Elgar.

Koschatzky, K. y R. Sternberg. 2000. "R\&D cooperation in innovation systems- some lessons from the European Regional Innovation Survey (ERIS)." European Planning Studies 8:487-501.

Kosckatzky, K. y A. Zenker. 1999. "The regional embeddedness of small manufacturing and service firms: regional networking as knowledge source for innovation?", Working Papers Firms and Regions R2/1999, Fraunhofer Institute systems and innovation research.

Lundvall, B. 1992a. National Systems of Innovation: Towards a Theory of Innovation and Interactive Learning. Londres: Pinter.

Lundvall, B. 1992b. "User-producer relationships, national sytems of innovation and internationalization". Pp. 45-67 en National systems of innovation, edited by B. Lundvall. Londres: Pinter.

Malerba, F. 2004. Sectoral Systems of Innovation. Concepts, Issues and Analyses of Six Major Sectors in Europe. Cambridge: Cambridge University Press.

Maskell, P. 2001. "Social capital, innovation and competitiveness". Pp. 11-123 in Social Capital: Critical perspectivas, edited by S. Baron, J. Field, y T. Schuller. Oxford: Oxford University Press.

Maskell, P. y A. Malmberg. 1999. "Localised learning and industrial competitiveness." Cambridge Journal of Economics 23:167-186.

Nelson, R .R. ed. 1993. National Innovation Systems: A Comparative Analysis. Oxford: Oxford University Press.

Nelson, R. R. 2008. "What enables rapid economic progress: What are the needed institutions?." Research Policy 37:1-11.

OECD. 2001. The Well-being of Nations. The Role of Human and Social Capital. París: OECD.

Olazaran, M., E. Albizu y B. Otero. 2009. Innovación en las pequeñas y medianas empresas industriales guipuzcoanas. Leioa: Ed. Servicio Editorial de la Universidad del País Vasco/Euskal Herriko Unibertsitatea. 
Orkestra. 2009. II Informe de Competitividad del País Vasco, Donostia-San Sebastián. Orkestra: Instituto Vasco de Competitividad.

Smallbone D., D. North y I. Vickers. 2003. "The role and characteristics of SMEs". Pp. 3-20 en Regional Innovation Policy for Small-medium Enterprises, edited by B. Asheim et al. Cheltenham: Edwar Elgar.

Storper, M. 1997. The Regional Economy. Nueva York: Guilford Press.

CRISTINA LAVÍA MARTÍNEZ es doctora en Sociología por la Universidad de Deusto y Profesora Titular del Departamento de Sociología en la UPV/EHU, especializada en metodología y análisis cuantitativo aplicados a ciencias sociales. Trabaja colaborando en proyectos de investigación sobre innovación y sistemas I+D así como también, más recientemente, en el desarrollo y análisis de indicadores sociales de diversa aplicación.

BEATRIZ OTERO GUTIÉRREZ es doctora en Sociología por la UPV-EHU y especialista en investigación aplicada y análisis de datos (Centro de Investigaciones Sociológicas). En la actualidad es investigadora en el instituto ILCLI de la UPV-EHU en el ámbito de los sistemas de I+D, la innovación y la organización.

MIKEL OLAZARAN RODRÍGUEZ es doctor en Sociología por la Universidad de Edimburgo y profesor titular en la UPV-EHU. Ha realizado proyectos y publicaciones en las áreas de ciencia y tecnología, organización, innovación y educación. Actualmente está trabajando en proyectos de investigación sobre sistemas regionales de innovación y sobre la formación profesional. Desde 2005 es decano del Colegio Oficial de Doctores/as y Licenciados/as en Ciencias Politicas y Sociología de Navarra.

ENEKA ALBIZU GALLASTEGI es doctor en Economía y profesor titular en la Universidad del País Vasco/Euskal Herriko Unibertsitatea. Sus intereses de investigación principales son "Innovación en pymes industriales" y "Recursos humanos e innovación". Es autor/coautor de numerosos artículos, libros, capítulos de libros publicados en editoriales de ámbito nacional e internacional.

RECIBIDO: 06/11/2009

ACEPTADO: 09/07/2010

Publicado on-line: 11/04/2010 